\title{
Autodependencia en los Cambios de Intensidad de la Experiencia Emocional Durante una Conversación Marital
}

\section{Selfdependency of Emotional Experience Intensity Changes During a Marital Conversation}

\author{
Juan Enrique Wilson Alcalde* \\ Juan Osvaldo Mejía Paredes
}

\begin{abstract}
Resumen
Treinta parejas conversaron durante veinte minutos intentando resolver un problema altamente conflictivo en su matrimonio. Cada persona evaluó la intensidad de su experiencia en siete emociones durante cada minuto de conversación. La conversación fue analizada en términos de transiciones desde la intensidad de la experiencia emocional en un minuto al siguiente y desde un nivel de intensidad a otro diferente. Los resultados para cada emoción muestran un patrón similar de auto dependencia. Este patrón puede ser caracterizado como una disposición a mantener la intensidad de la experiencia emocional de un minuto al siguiente y como una disposición a que los cambios en el nivel de intensidad ocurran sólo en la forma de incrementos o decrementos leves.
\end{abstract}

Palabras Claves: Emoción, Interacción, Marital

\section{Abstract}

Thirty couples talked during 20 minutes attempting to solve a highly conflictive problem in their marriage. Each subject rated his/her experience about seven specific emotions during every minute of the conversation. The conversation was analyzed in terms of transitions from the emotional experience intensity on one minute to the next and from one level of intensity to another. The results for each emotion showed a similar pattern of self-dependency. This pattern can be characterized as a disposition to maintain the emotional experience intensity from one minute to the next and as a disposition for changes on levels of intensity to appear only as slight increases or decreases.

Key Words: Emotion, Interaction, Marital

* Académico Departamento de Psicología, Universidad de Chile.e-mail: jewilson@uchile.cl 
Instancias de comunicación marital proveen de un contexto particularmente ventajoso para el estudio de las emociones. Tales situaciones permiten explorar los aspectos dinámicos (temporales) y relacionales (interaccionales) de la afectividad, bajo una situación cotidiana y socialmente muy significativa. Esto contrasta con un estudio de la emoción en el cual el individuo emite una respuesta discreta ante una imagen, generalmente, estática, predefinida por el investigador y de escasa relevancia personal para el participante.

Los estudios en afectividad que se basan en el primer paradigma de investigación mencionado, más naturalístico, son, no obstante, relativamente escasos. Esto es así, principalmente, por las exigencias que impone la tarea a investigadores y participantes. Así, en lugar de preguntar a las parejas cómo se comunican (a través de un cuestionario o entrevista), se les solicita que concretamente se comuniquen. Es necesario que la conversación se refiera a un tema que sea verdaderamente relevante para ellos en su particular relación. A su vez, la interacción debe ser grabada, de modo que posteriormente pueda ser observada por los propios cónyuges (dando lugar a un auto reporte de su experiencia emocional) y/o por observadores externos (dando lugar a la codificación de la conducta emocional de los cónyuges). Se requiere que el registro de los datos, proporcionados por los participantes o los observadores externos, respete la dinámica o temporalidad de los eventos en la conversación. Finalmente, es necesario analizar estos datos de modo tal que pueda develarse la posible estructura de la interacción, es decir, que su naturaleza relacional quede de manifiesto.

Esta metodología ha permitido evidenciar que, en comparación con las parejas bien avenidas, las parejas insatisfechas exhiben un mayor grado de interdependencia afectiva durante la comunicación (para una revisión, ver a Gottman, 1998; Weiss \& Heyman, 1990). Esto significa: 1) una mayor propensión a responder con una conducta emocional negativa ante una conducta emocional negativa del cónyuge (e.g., Margolin \& Wampold, 1981; Burman, Margolin, \& John, 1993), 2) una mayor propensión a experimentar conjuntamente o casi simultáneamente sentimientos negativos (e.g., Levenson \& Gottman, 1983; Levenson, Carstensen, \& Gottman, 1994), 3) una mayor propensión a evaluar negativamente los mensajes negativos y neutros del cónyuge y a responder en consonancia con esa evaluación (Notarius, Benson, Sloane, \& Vanzetti, 1989) y 4) un mayor grado de correspondencia temporal en la activación fisiológica (Levenson \& Gottman, 1983).

La interdependencia en la afectividad de los cónyuges ha sido establecida en estos estudios mediante el análisis de la secuencia interaccional. Ello implica, en primer lugar, dividir el flujo de la conversación en unidades de observación que pueden ser eventos (e.g., turnos de habla) o intervalos (e.g., cada 10 segundos). A su vez, estas unidades son caracterizadas asignándoles una conducta emocional discreta (e.g., durante el turno de habla " $X$ " del marido, ¿su conducta emocional fue positiva, negativa o neutral?) o un puntaje en una escala afectiva (e.g., durante el intervalo " $X$ ", y de acuerdo a una escala que va de muy positiva a muy negativa, ¿cuál fue la experiencia emocional del marido?). Luego, estas unidades son transformadas en transiciones desde un evento (o intervalo) antecedente dado hacia un evento consecuente. Para estimar el grado de dependencia del consecuente respecto de un antecedente en particular, se compara la probabilidad de que ocurra el consecuente dado ese antecedente (su probabilidad condicional) con la probabilidad de que ocurra el consecuente dada cualquier circunstancia antecedente (su probabilidad incondicional). Se dice 
que existe dependencia cuando la probabilidad condicional del evento consecuente es significativamente mayor que su probabilidad incondicional; en otras palabras, el investigador ha aumentado su habilidad para predecir la conducta de un sujeto a partir del conocimiento de la conducta previa del cónyuge (ver Bakeman \& Gottman, 1986). Por ejemplo, Margolin \& Wampold (1981) señalan que existe reciprocidad negativa cuando la probabilidad de que un individuo responda negativamente ante la conducta emocional negativa de su cónyuge, es mayor que la probabilidad de que ese individuo emita una conducta emocional negativa ante cualquier conducta emocional antecedente de su cónyuge.

Tal como lo hemos descrito, los estudios maritales realizados hasta ahora se han focalizado en la influencia mutua de los cónyuges. Sin embargo, es posible que la ocurrencia de un cierto evento psicológico no esté controlada necesaria o exclusivamente por el medio externo, sino que también esté en función de eventos psicológicos proveídos por el propio sujeto. Por lo tanto, un evento psicológico no sólo sería una respuesta al medio, sino que además constituiría un estímulo discriminativo para otros eventos psicológicos en el individuo. El estudio que nosotros realizamos se inserta en esta última línea de pensamiento. En concreto, el objetivo es estimar si la intensidad de la experiencia emocional de un sujeto en un momento de la conversación marital depende de la intensidad de su experiencia emocional en un instante previo de la conversación. Con ello no desconocemos la posible influencia ejercida en el sujeto por el contexto interaccional en el cual se encuentra inserto, sino que, tan sólo, el foco de este estudio no está puesto en dicha influencia externa.

\section{Método}

\section{Participantes}

Matrimonios de la ciudad de Santiago fueron contactados, principalmente, a través de cartas enviadas a los centros de padres de varios colegios. En estas cartas se informó acerca de un estudio en comunicación y ajuste marital que estaban llevando a cabo en el Departamento de Psicología de la Universidad de Chile. Las parejas interesadas contactaron telefónicamente al investigador principal. Durante esta conversación se terminaron de aclarar las dudas respecto de la participación en el estudio y se acordó con las parejas las fechas y horarios de participación.

Los resultados reportados en este artículo corresponden a las primeras 30 parejas que, una vez plenamente informadas, accedieron a participar. Las parejas llevaban casadas un promedio de 13.7 años (rango de 40 a $1 ; \mathrm{s}=8.25$ ) y tenían como promedio 2.17 hijos (rango de 4 a $0 ; \mathrm{s}=1.05$ ). Los sujetos tenían un puntaje promedio de Ajuste Marital (Short Marital Adjustment Test; Locke \& Wallace, 1959) de 101,2 $(\mathrm{Md}=108,5 ; \mathrm{S}=28,40 ; \mathrm{y}$ Rango entre 17 y 151 puntos). El puntaje total posible en el SMAT va de 2 a 158 puntos, considerándose habitualmente como maritalmente ajustado al individuo que obtiene sobre 100 puntos. En cuanto al nivel educacional de las personas en la muestra, el 3.3\% tenía educación básica completa, el $15 \%$ educación media incompleta, el $18.3 \%$ educación media completa, el $1.7 \%$ educación técnica incompleta, el $36.7 \%$ educación técnica completa, el $13.3 \%$ educación universitaria incompleta, y el 11.7\% educación universitaria completa. 


\section{Procedimiento}

Sesión de Cuestionarios

Todo el estudio se llevo a cabo en el Centro de Psicología Aplicada (CAPS) de la Facultad de Ciencias Sociales de la Universidad de Chile. Durante la primera reunión se realizó una breve entrevista con la pareja y a continuación procedieron a llenar los cuestionarios en habitaciones separadas. Las parejas llenaron: a) un inventario de variables demográficas (e.g., años de casados, nivel educacional, número de hijos), b) un cuestionario de comunicación, c) un Cuestionario de Áreas de Conflicto Marital (CUCOM), y d) el Short Marital Adjustment Test (SMAT; Locke \& Wallace, 1959).

El CUCOM fue especialmente creado en esta investigación para que los cónyuges pudieran evaluar, de acuerdo a una escala de 0 (nada) a 4 (mucho), cuánto conflicto en su relación marital estaba asociado a cada una de las 109 situaciones presentadas en el instrumento. Los resultados en este instrumento fueron usados en una segunda sesión para definir con la pareja un tema a ser conversado por ellos. La correlación entre el SMAT y el CUCOM fue de - 0.56 (a mayor ajuste marital, menor conflicto asociado a situaciones matrimoniales), lo cual sugiere que las respuestas entregadas al CUCOM funcionan adecuadamente como un indicador del grado de conflicto presente en la relación marital y, entonces, puede ser usado para identificar áreas de discusión.

ElSMAT es uno de los instrumentos para evaluar el ajuste marital más utilizados en la investigación. Consta de 15 itemes, 8 de los cuales evalúan el grado general de acuerdo en los siguientes temas: finanzas familiares, recreación, demostraciones de afecto, amistades, relaciones sexuales, convencionalismos sociales, filosofía de la vida y trato con los parientes políticos. Los restantes 7 itemes cubren el grado general de satisfacción matrimonial, preferencias de cada uno respecto del uso del tiempo libre, el grado de actividades compartidas fuera del hogar, arrepentimientos respecto a haberse casado (con el cónyuge en particular y también en general), confianza en el cónyuge y la forma típica de término de una discusión marital. La correlación entre el ajuste marital masculino y femenino fue de 0.66 , lo que implica que, en general, los individuos que ven más problemas y están más insatisfechos con su matrimonio están casados con personas que opinan y sienten lo mismo.

Aproximadamente una semana después de la sesión de cuestionarios, cada pareja regresó al Centro para participar en tres sesiones de actividad diferentes: Sesión de Conversación, Sesión de Reporte Global de la Conversación y, Sesión de Evaluación del Video de la Conversación.

\section{Sesión de Conversación}

Cada pareja sostuvo una conversación, durante la cual se registró su actividad cardiaca y se obtuvo un video. Se planteó a la pareja que el objetivo de dicha conversación era que hicieran su mejor intento por resolver un tema que fuera altamente conflictivo en su relación matrimonial. Con ese requisito en mente, los cónyuges seleccionaron como tema de conversación una de las situaciones entre aquellas que en la sesión previa ambos habían marcado como las más altamente conflictivas en el CUCOM. Después de esta elección, la pareja se dirigió a otra habitación para conversar el tema. En esta sala habían dos sillas situadas frente a frente, dos cámaras (cada una enfocada a cada silla), dos amplificadores (cada uno situado en la base de cada silla), una mesita con cables y electrodos, y un citófono. Se le colocaron tres electrodos a cada cónyuge, cuyas señales fueron recogidas por los res- 
pectivos amplificadores y enviadas para su almacenamiento a un computador ubicado en la sala contigua. En seguida, se indicó a la pareja que serían dejados a solas en esa sala durante 25 minutos y que, a partir de ahí, sostuvieran una conversación durante los primeros 5 minutos respecto de lo que habían hecho en el día (i.e., conversación baja en conflicto) para luego conversar el tema conflictivo seleccionado durante los 20 minutos restantes. Se les dijo que no se ocuparan de tomar el tiempo, sino que sólo prestaran atención al sonido del citófono que les indicaría el momento de inicio y término de las conversaciones. Dadas estas instrucciones, se encendieron las cámaras y se les dejó a solas en la habitación. Inmediatamente después, se emitió el primer sonido del citófono y, simultáneamente, se dio comienzo al registro de la actividad cardiaca de cada cónyuge. Los sonidos del citófono quedaron registrados en el video de la conversación, lo cual hace posible sincronizar las conductas conversacionales de los cónyuges con el registro de su actividad cardiaca.

\section{Sesión de Reporte Global de la Conversación}

Terminada su conversación, cada cónyuge se encaminó a una sala independiente para completar una hoja titulada "Reporte Global de la Conversación". Ayudados sólo por su recuerdo, en esa hoja cada cónyuge debía indicar cómo se sintió, en general, durante la conversación. De modo similar, cada persona debía reportar ahí cómo creía que se había sentido su pareja durante la conversación. Para hacer ambas evaluaciones, la persona tenía escrita en su hoja una escala que iba de 0 (nada) a 3 (muy), una columna a la izquierda con el encabezado "Yo me sentí" y una columna a la derecha encabezada "Ella se sintió" o "El se sintió", según fuera el caso. Bajo cada encabezamiento había un mismo listado de siete categorías emocionales, y al lado de cada categoría se veían cuatro casilleros para que la persona marcara, según la escala, la intensidad de su emoción o la del cónyuge. Cada categoría emocional estaba constituida por cinco adjetivos afectivos (un estudio piloto mostró que los cinco adjetivos tenían un significado próximo o común), uno de los cuales aparecía en mayúsculas para titular a la categoría. Las categorías emocionales fueron: ENOJADO (enrabiado, exasperado, fastidiado o irritado), TRISTE (solitario, abandonado, débil o desesperanzado), TEMEROSO (nervioso, angustiado, preocupado o inseguro), INDIFERENTE (desinteresado, insensible, apático o frío), ALEGRE (excitado, animado, entusiasta o chispeante), CARIÑOSO (enternecido, afectuoso, querendón o amoroso), y TRANQUILO (relajado, sereno, apacible o calmado). Dos fueron los objetivos de usar este Reporte Global de la Conversación. Primero, este reporte era similar al que debían hacer las parejas a continuación, en la Sesión de Evaluación del Video de la Conversación, con la diferencia que, como veremos, esta segunda tarea era notablemente más exigente. Por lo tanto, realizar el Reporte Global de la Conversación le permitía a los cónyuges familiarizarse previamente con el tipo de instrumento que usarían al evaluar el video (e.g., las categorías emocionales y la escala). Segundo, las respuestas al Reporte Global miden el recuerdo de los cónyuges de la conversación, en tanto que la tarea de Evaluación del Video mide más bien su percepción (nos interesa examinar el nivel de congruencia entre el recuerdo y la percepción de la conversación, pero este análisis será reportado en un próximo estudio). 
Sesión de Evaluación del Video de la Conversación

Inmediatamente terminado el reporte global, los cónyuges procedieron a ver el video de la conversación. La señal del video era enviada desde el exterior de modo que llegara simultáneamente al monitor que tenía enfrente cada cónyuge en su sala. Sin embargo, y para que fuera una situación similar a lo que ocurre en una conversación, cada persona sólo se escuchaba a sí misma en tanto que veía y escuchaba a su pareja. El video fue pausado a intervalos de 1 minuto para que los cónyuges respondieran cómo se habían sentido y cómo creían que se había sentido su pareja durante cada uno de estos momentos de la conversación. Para responder, los sujetos hacían marcas en hojas similares a la del reporte global, con la sola diferencia de que ahora contaban con veinte hojas (una para cada intervalo) apiladas en secuencia y tituladas cada una con el número de intervalo respectivo. Por lo tanto, en cada intervalo los sujetos reportaban la intensidad para cada emoción sentida y percibida de acuerdo con la escala de " 0 " (nada) a "3" (muy). Para ello disponían de 1 minuto como límite, luego del cual se soltaba la pausa y se les mostraba el siguiente intervalo (y así de ahí en adelante hasta completar los veinte minutos de conversación). Por lo tanto, el tiempo total de esta tarea de evaluación del video fue de 40 minutos. El reporte de sentimientos que realizaron los sujetos en cada intervalo constituye las secuencias que nos interesa analizar en este estudio.

\section{Resultados}

\section{Validez del Reporte de Sentimientos}

Previamente a describir el análisis de secuencias de los sentimientos a lo largo de la conversación, es importante señalar que contamos con datos que avalan la validez del procedimiento utilizado para recabar estos datos. Así, la experiencia subjetiva emocional de los participantes correlacionó con a) la percepción del cónyuge de dichos sentimientos, b) la percepción de observadores externos de las emociones desplegadas durante la conversación, c) los puntajes en el SMAT , d) el Reporte Global de los Sentimientos $\mathrm{y}, \mathrm{e}$ ) los puntajes en el CUCOM.

\section{Análisis de Transiciones Temporales}

Llamaremos al nivel de intensidad de la experiencia emocional en un intervalo cualquiera de la interacción el evento antecedente y al nivel reportado en el intervalo siguiente el evento consecuente. El movimiento desde un evento antecedente (a) a un evento consecuente (c) se conoce como transición $(\mathrm{a} \otimes \mathrm{C})$. Se utiliza la palabra "Lag" para indicar este desplazamiento en el tiempo. Si el evento consecuente ocurre inmediatamente después del evento antecedente se habla de una transición Lag 1, si ocurre después de un evento interviniente, de Lag 2, etc. Por ejemplo, imagine la siguiente secuencia de reportes emocionales:

$\begin{array}{llllllll}0 & 2 & 2 & 1 & 3 & 0 & 2 & 1\end{array}$

Para identificar las transiciones Lag1, usamos una "ventana de tiempo móvil" que se desliza a través de los eventos contiguos de la secuencia en la siguiente manera:
$(0$
2)
2
1
3
0
21

para identificar la primera transición,

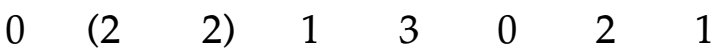

para identificar la segunda transición,

$\begin{array}{llllllll}0 & 2 & (2 & 1) & 3 & 0 & 2 & 1\end{array}$

para identificar la tercera transición, etc. 
En este caso la primera transición (primera posición de la ventana) indica que la persona pasó de estar nada emocionada en un minuto dado a estar bastante emocionada en el minuto siguiente (0® 2); la segunda transición indica que la persona se mantuvo bastante emocionada de un minuto al siguiente (2® 2); la tercera posición indica que la persona transitó de estar bastante emocionada a ligeramente emocionada en el minuto siguiente (2® 1). En la secuencia dada como ejemplo, la transición (0®2) ocurrió dos veces, la transición ( $2 \circledast 2)$ ocurrió una sola vez, la $(2 \circledast 1)$ ocurrió dos veces, la $(1 \circledast 3)$ ocurrió una vez, etc.

En nuestros estudio analizamos sólo transiciones Lag1, lo que da lugar a 16 diferentes transiciones posibles (4 niveles de intensidad en el minuto antecedente $x 4$ niveles de intensidad en el minuto siguiente). Como la conversación marital duró 20 minutos, cada individuo aportó 19 transiciones por emoción. Para llevar a cabo el análisis, estos datos fueron sumados a través de los 60 sujetos, dando origen a un total de 1140 transiciones por emoción. La figura 1 muestra para la emoción "enojo" la frecuencia observada de cada una de las 16 transiciones posibles. La matriz se formó designando a las filas como eventos antecedentes (Lag 0 ) y a las columnas como eventos consecuentes (Lag 1). La última columna a la derecha $(\mathrm{f}(\mathrm{a})$ ) muestra los totales para cada fila, es decir, la frecuencia total para las transiciones que comenzaron con un cierto evento antecedente. Por ejemplo, el evento " 0 " (nada enojado) ocurrió como antecedente en 538 transiciones y el evento " 1 " (un poco enojado) en 391 transiciones. A su vez, la última fila $(\mathrm{f}(\mathrm{c})$ ) señala los totales para cada columna, es decir, la frecuencia total de las transiciones que terminaron con un cierto evento consecuente. Por ejemplo, el evento " 0 " ocurrió como consecuente en 527 transiciones y el evento " 3 " (muy enojado) en 47 transiciones. La entrada en la fila "CERO" y la columna "CERO" es el número de veces en la secuencia que el evento " 0 " siguió al evento " 0 " $(\mathrm{f}(0 / 0))$; la entrada en la fila "CERO" y la columna "TRES" es el número de veces en la secuencia que el evento " 3 " siguió al evento " 0 " $(\mathrm{f}(3 / 0))$. Comparando estas dos celdillas, se ve claramente que la transición " $0 / 0$ " fue mucho más frecuente que la transición " $3 / 0$ ", pues la primera ocurrió en un $35,3 \%$ de los casos $(402 / 1140 \times 100)$ y la segunda sólo en un $0.26 \%$ de los casos (3/ 1140x100).

La figura 2 es la matriz de probabilidades transicionales para "enojo". Cada celdilla representa la proporción/probabilidad ob-

Figura 1: MATRIZ DE FRECUENCIAS TRANSICIONALES PARA ENOJO

\begin{tabular}{|c|c|c|c|c|c|c|}
\hline \multirow{7}{*}{ 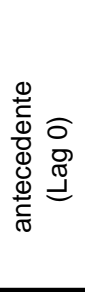 } & & \multicolumn{4}{|c|}{ consecuente (Lag 1) } & \multirow{3}{*}{$\begin{array}{r}f(a) \\
538\end{array}$} \\
\hline & \multirow{6}{*}{$\begin{array}{c}\text { CERO } \\
\text { UNO } \\
\text { DOS } \\
\text { TRES } \\
f(c)\end{array}$} & CERO & UNO & DOS & TRES & \\
\hline & & 402 & 112 & 21 & 3 & \\
\hline & & 109 & 201 & 73 & 8 & 391 \\
\hline & & 12 & 69 & 75 & $\overline{12}$ & 168 \\
\hline & & 4 & 9 & 6 & 24 & 43 \\
\hline & & 527 & 391 & 175 & 47 & 1140 \\
\hline
\end{tabular}


servada de un evento consecuente dado un antecedente particular (p(c/a)) o, lo que es lo mismo, la probabilidad condicional de un evento. Debe observarse que una probabilidad transicional no representa la frecuencia con que ocurrió una determinada transición respecto del total de transiciones, esta última sería la probabilidad simple de una transición. De hecho, la probabilidad transicional se calcula dividiendo la frecuencia de una transición (una celdilla en la tabla de frecuencias) no por el total de transiciones, sino que por el total de transiciones que comenzaron con ese mismo antecedente (la suma total de celdillas en esa misma hilera of(a)). Por ejemplo, la probabilidad transicional para " $3 / 3$ " es de $0.56(24 / 43)$, en tanto que la probabili- dad simple para esta misma transición es sólo de $0.02(24 / 1140)$. Siguiendo con las otras probabilidades transicionales para el 3 como antecedente se observa que la $\mathrm{p}(2 / 3)=0.14$, $p(1 / 3)=0.21$ y $p(0 / 3)=0.09$. Por lo tanto, las probabilidades de las transiciones que comenzaron con un mismo antecedente suman 1. Comparando todas estas probabilidades transicionales se observa que la consecuencia más probable del evento " 3 " es el propio evento " 3 ". En términos más concretos, lo que estas probabilidades nos dicen es que si se está muy enojado en un momento, lo más probable es que se continúe estando muy enojado en el minuto siguiente.

Lo que las probabilidades transicionales no nos dicen, sin embargo, es si la aparición

Figura 2: MATRIZ DE PROBABILIDADES TRANSICIONALES PARA ENOJO

\begin{tabular}{|c|c|c|c|c|c|c|c|}
\hline \multirow{8}{*}{ 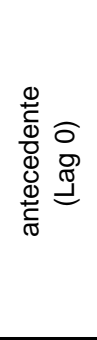 } & \multirow{6}{*}{$\begin{array}{c}\text { CERO } \\
\text { UNO } \\
\text { DOS } \\
\text { TRES }\end{array}$} & & \multicolumn{3}{|c|}{ consecuente (Lag 1) } & & \multirow[b]{2}{*}{$P(a)$} \\
\hline & & CERO & UNO & DOS & TRES & \multirow{6}{*}{$\begin{array}{c}f(a) \\
538 \\
391 \\
168 \\
43 \\
1140\end{array}$} & \\
\hline & & 0.75 & 0.21 & 0.04 & 0.01 & & 0.47 \\
\hline & & 0.28 & 0.51 & 0.19 & 0.02 & & 0.34 \\
\hline & & 0.07 & 0.41 & 0.45 & 0.07 & & 0.15 \\
\hline & & 0.09 & 0.21 & 0.14 & 0.56 & & 0.04 \\
\hline & $f(c)$ & 527 & 391 & 175 & 47 & & \\
\hline & $P(c)$ & 0.46 & 0.34 & 0.15 & 0.04 & & \\
\hline
\end{tabular}

del evento consecuente depende del evento antecedente. Para estimar esa dependencia, la probabilidad condicional del consecuente (su ocurrencia dado un cierto antecedente), debe ser significativamente diferente de su probabilidad incondicional (su ocurrencia dado cualquier antecedente). Esta comparación es necesaria dado que la probabilidad de una transición puede ser relativamente alta como mero producto de la alta frecuencia con que ocurrieron en la secuencia los eventos que componen esta transición. En otras palabras, la probabilidad observada de una transición debe ser significativamente diferente de lo que se esperaría por azar. Para determinar la dependencia entre dos eventos en una transición, compararemos su probabilidad observada con su probabilidad esperada mediante la aproximación normal (puntaje z) a la distribución binomial. El puntaje z para cada transición se calcula de la siguiente forma:

$$
Z=\frac{p(c / a)-p(c)}{\sqrt{\frac{p(c)[1-p(c)][1-p(a)]}{N p(a)}}}
$$

donde $\mathrm{p}(\mathrm{c} / \mathrm{a})$ es la probabilidad observada de la transición, $\mathrm{p}(\mathrm{c})$ es la probabilidad incondicional del consecuente $(\mathrm{f}(\mathrm{c}) / \mathrm{N})$; $\mathrm{p}(\mathrm{a})$ es la probabilidad del antecedente $(\mathrm{f}(\mathrm{a}) / \mathrm{N})$ 
y $\mathrm{N}$ es el número total de transiciones. Valores $\mathrm{z}$ superiores $\mathrm{a}+1.96$ indican que la ocurrencia del evento antecedente aumenta fuertemente la probabilidad del evento consecuente. Valores z inferiores a -1.96 indican que la presencia del evento antecedente disminuye drásticamente la probabilidad de ocurrencia del evento consecuente. Finalmente, valores entre $-1.96 \mathrm{y}+1.96$ indican que el evento antecedente tiene poco o ningún impacto en la aparición del evento consecuente. A modo de ilustración mostraremos el cálculo de $\mathrm{z}$ para la transición del 1 como consecuente del $2(\mathrm{z}(1 / 2))$.

$$
\begin{gathered}
Z=\frac{p(1 / 2)-p(1)}{\sqrt{\frac{p(1)[1-p(1)][1-p(2)]}{N p(2)}}} \\
Z=\frac{0.41-0.34}{\sqrt{\frac{0.34[1-0.34][1-0.15]}{1140 x 0.15}}} \\
Z=2.00
\end{gathered}
$$

Para este caso la probabilidad observada del evento 1 dado el antecedente 2 es significativamente mayor que su probabilidad esperada: es decir, la presencia del evento 2 aumenta fuertemente la probabilidad de aparición del evento 1. Los valores z para las otras transiciones que comienzan con el evento 2 son: $z(0 / 2)=-11.00 ; z(2 / 2)=11.00$; $z(3 / 2)=2.13$. En resumen, estar bastante enojado aumenta significativamente la probabilidad de estar bastante, un poco y muy enojado al minuto siguiente, y disminuye significativamente la probabilidad de estar nada enojado al minuto siguiente.

La tabla 1 muestra la matriz de transiciones temporales para todas las emociones. Se muestran los puntajes $\mathrm{z}$ sólo para las transiciones significativas, probables (se subrayan los puntajes $\mathrm{z}$ positivos) e improbables (puntajes z negativos). Lo que más destaca en la tabla es que cada emoción muestra un patrón similar de resultados: cualquiera sea el nivel de intensidad de la experiencia emocional en un momento dado (nada, un poco, bastante o muy emocionado), este nivel tiende a mantenerse en el minuto siguiente.

\section{Análisis de Transiciones de Estados}

En el análisis anterior cada reporte de la experiencia emocional representaba un intervalo de tiempo en la secuencia, en este caso, de un minuto. El análisis que ahora realizamos parte por construir una secuencia de eventos a partir de los datos de esta secuencia temporal. Al construir esta secuencia de eventos a partir del registro original, se reduce el número de datos en la secuencia y cada uno de éstos pasa a representar un estado de intensidad emocional diferente. A modo de ejemplo, presentamos un tramo de la secuencia temporal original y su reducción a una secuencia de estados emocionales.

Secuencia temporal:

$\begin{array}{llllllll}0 & 2 & 2 & 1 & 1 & 0 & 3 & 3\end{array}$

Secuencia de estados:

$\begin{array}{lllll}0 & 2 & 1 & 0 & 3\end{array}$

El análisis de esta secuencia de estados nos permitirá ver cómo se produce el cambio en el nivel de activación emocional, independientemente del momento en que éste se produce. Llamaremos incrementos " $\mathrm{I}$ " a las transiciones desde un estado de activación dado a un estado de mayor activación, y decrementos " $\mathrm{D}$ " a las transiciones hacia un estado de menor activación. A su vez, tanto los incrementos como los decrementos pueden 


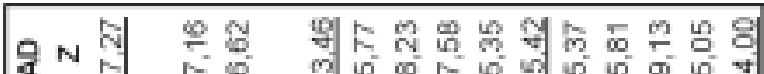
过

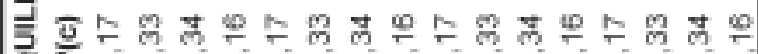

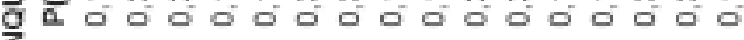

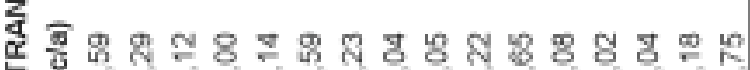

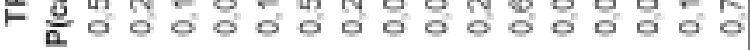

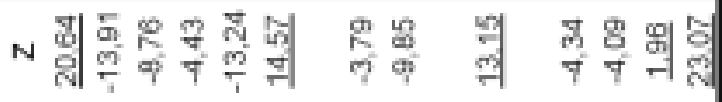

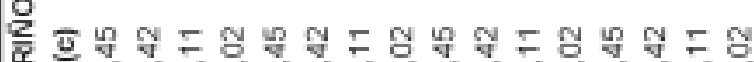

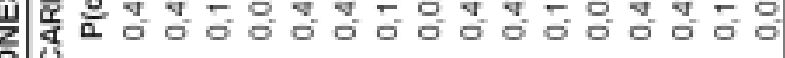
可的舟

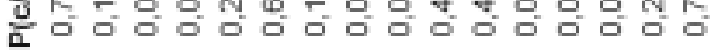

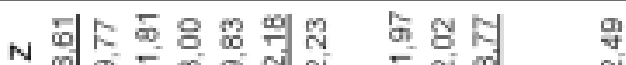

ㄸำ

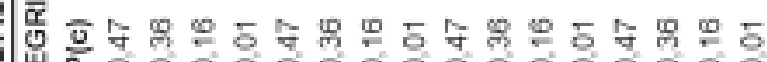

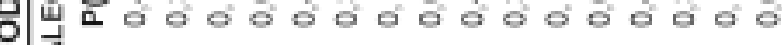
ष

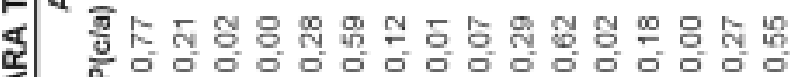

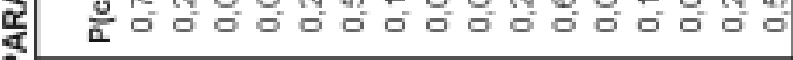

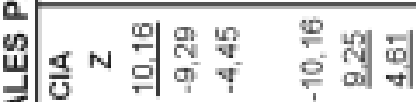

\$ิ

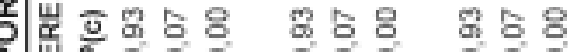

语

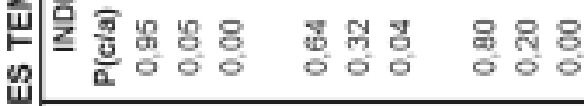

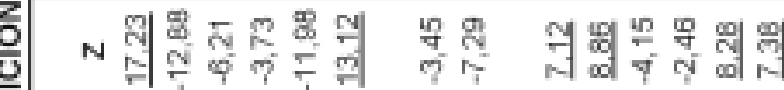

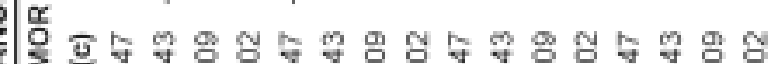

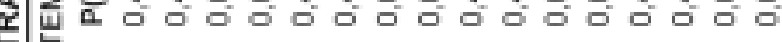

幽 要品 N

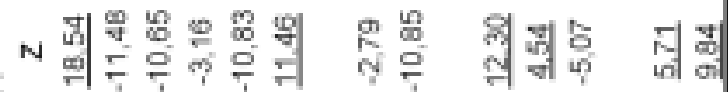

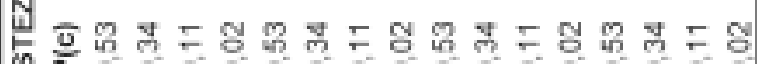

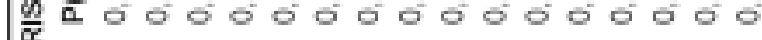

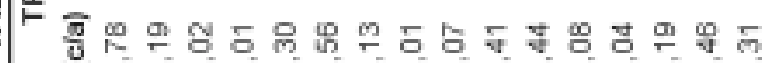

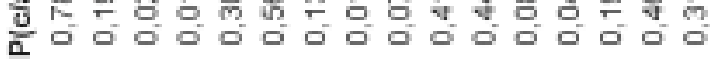

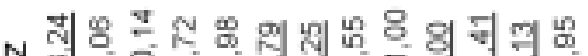

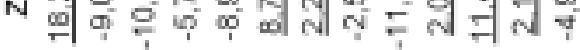

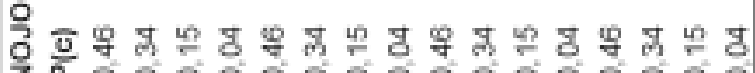

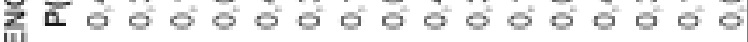

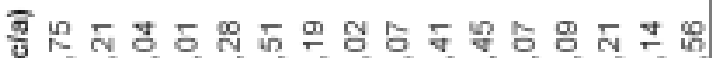

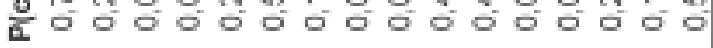

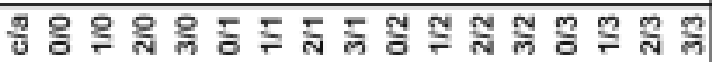

咹

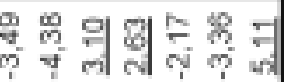

वें

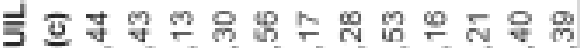

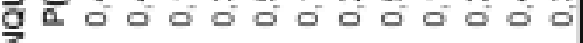
依

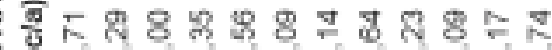
응ㅇㅇㅇㅇㅇ

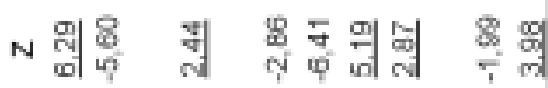

을

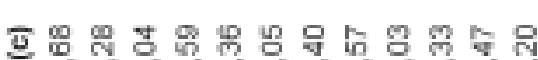

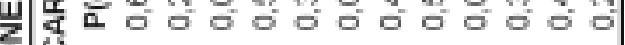

읭

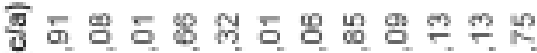

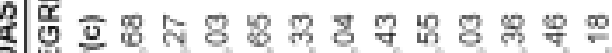

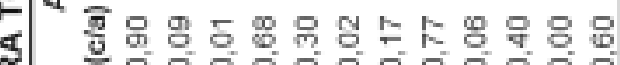

$\triangle N$ is

要

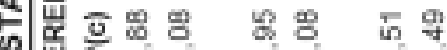

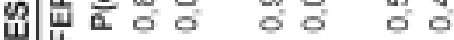

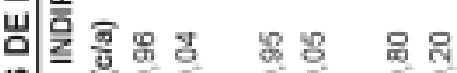

行

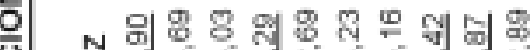

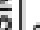

:

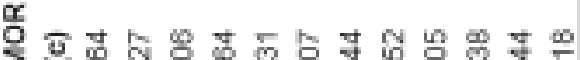

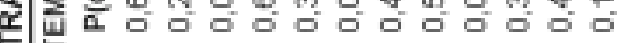

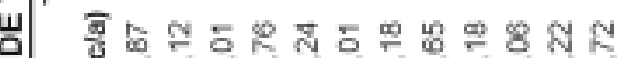

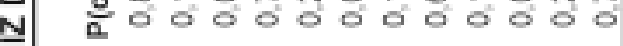

항

3

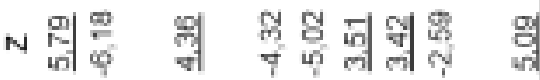

is

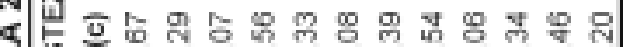

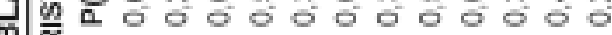
罢金

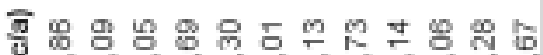

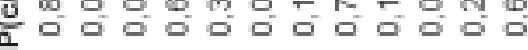

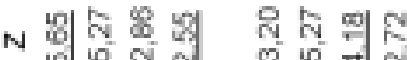

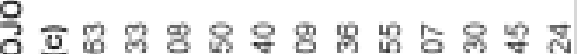

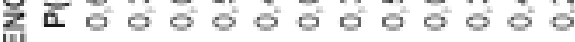

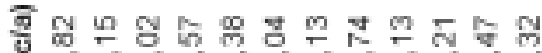

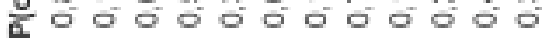

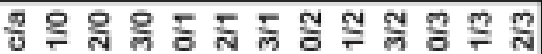


Ocurrir de forma leve " $L$ ", moderada " $M$ " o fuerte " $\mathrm{F}$ ", dependiendo de la magnitud del cambio en intensidad de la experiencia emocional. La figura 3 muestra la nomenclatura para todas las transiciones de estados posibles. Por ejemplo, las transiciones "1/
0 ", “2/1", “3/2" constituyen todas un tipo particular de incremento leve "IL": cambiar de estar nada emocionado a estar un poco emocionado, de un poco a bastante, y de bastante a muy, respectivamente.

Fig 3: Nomenclatura para Transiciones de Estados

Consecuente

\begin{tabular}{|c|c|c|c|c|}
\hline & CERO & UNO & DOS & TRES \\
\hline CERO & -- & $\mathrm{IL}$ & $\mathrm{IM}$ & $\mathrm{IF}$ \\
\hline UNO & DL & -- & IL & IM \\
\hline DOS & DM & DL & -- & IL \\
\hline TRES & DF & $\mathrm{DM}$ & DL & - \\
\hline
\end{tabular}

El cálculo y la evaluación de los puntajes z para estas transiciones de estado es similar al realizado para las transiciones temporales, con la excepción del cálculo de la probabilidad del consecuente. Dado que ahora un mismo estado no puede ser antecedente y consecuente a la vez en una transición, la probabilidad de un estado consecuente es calculada dividiendo su frecuencia, no por el número total de estados codificados, sino por el número de veces que pudiera ocurrir después del estado antecedente. Entonces, $p(c)=f(c) /(N-f(a))$.

La tabla 2 muestra la matriz de transiciones de estado para todas las emociones. Nótese que, salvo la emoción "indiferencia", se observa un patrón similar de resultados a través de todas las emociones. Este patrón se caracteriza por: a) una propensión a experimentar incrementos y decrementos leves $y, b)$ una indisposición a experimentar incrementos y decrementos tanto moderados como fuertes. Una excepción en el patrón de incrementos leves, que se aprecia en todas las emociones, lo constituye la transición del estado de actividad " 1 " a " 2 ", puesto que la probabilidad de ésta no es significativamente mayor ni menor a la esperada.
Los resultados más importantes de las tablas 1 y 2 son representados gráficamente a continuación. De esta manera, sólo se incluyen en el diagrama las transiciones temporales y de estados significativamente probables e improbables que, además, aparecen consistentemente a través de las emociones. Las flechas curvas en el diagrama representan la dependencia entre el nivel de activación emocional en un minuto dado y el nivel en el minuto siguiente. Como estas flechas salen y entran al mismo nivel de activación, esto indica la propensión a mantener el grado de activación de un momento a otro. Las flechas rectas representan la dependencia entre dos niveles de activación diferentes. Cuando estas flechas son continuas, la dependencia puede ser caracterizada como facilitación del tránsito de un nivel de actividad a otro. Es decir, un estado aumenta significativamente la probabilidad de aparición del otro estado. A su vez, cuando las flechas aparecen tarjadas, esto indica inhibición del tránsito de un nivel de actividad a otro. Es decir, un estado disminuye significativamente la probabilidad de aparición del otro estado. Tomando la emoción "enojo" como ejemplo, las flechas que entran y salen de " 3 " indican que la probabilidad 
de estar muy enojado aumenta significativamente cuando la persona ha estado muy enojada en el minuto anterior (flecha curva) o viene de estar bastante enoja- da (flecha de "2" a "3"), y disminuye significativamente si la persona estaba ligeramente enojada (flecha de " 1 " a " 3 ") o no lo estaba en absoluto (flecha de " 0 " a " 3 ").

\section{DIAGRAMA MODELO DE LAS TRANSICIONES TEMPORALES Y DE ESTADOS}

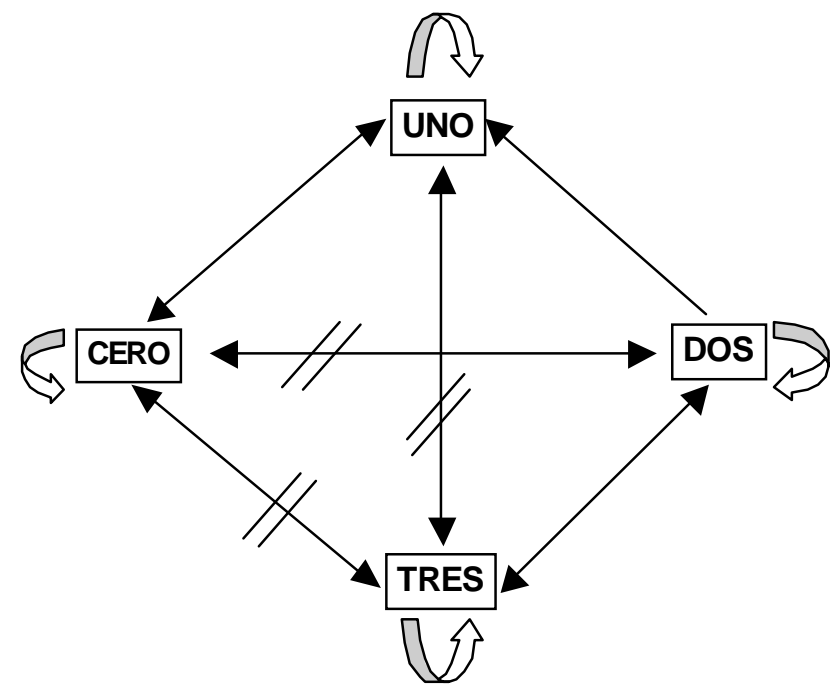

\section{Discusión y conclusiones}

Varios estudios maritales han mostrado que es posible predecir el funcionamiento emocional (experiencial, fisiológico o conductual) de un individuo a partir del funcionamiento emocional de su cónyuge durante la interacción. Aunque no ha sido el objetivo de estos estudios, teóricamente estos cambios también pueden ser predichos a partir de la propia dinámica interna del sujeto durante la interacción marital. El objetivo de este trabajo fue explorar la potencia de este segundo modelo de predicción. En particular, se estimó el grado en que la magnitud de la experiencia emocional del individuo depende de la magnitud de su propia experiencia emocional previa durante la conversación marital. Para afirmar que hubo tal dependencia, la probabilidad de tener una experiencia emocional de una cierta magni- tud en cualquier momento de la conversación debe ser significativamente diferente (i.e., menor o mayor) de su probabilidad dada una cierta magnitud precedente.

Los resultados evidencian un alto grado de auto dependencia en la magnitud de la experiencia emocional. En primer lugar, cualquiera sea la emoción y la magnitud de su vivencia, el sujeto está predispuesto a mantener este nivel de activación de un minuto a otro. En segundo lugar, y cualquiera sea la emoción, cuando ocurre un cambio en la intensidad de la experiencia, cuál sea el nivel de intensidad alcanzado depende de aquel que le antecedió. En particular, el sujeto está predispuesto a transitar desde un cierto nivel de activación a aquel más próximo o, en otras palabras, existe una propensión a que ocurran cambios graduales en la magnitud de la experiencia emocional: incremen- 
tos o decrementos leves. Es interesante que, además, existe una alta indisposición a experimentar cambios bruscos de intensidad, sean éstos incrementos o decrementos.

Estas predisposiciones e indisposiciones a transitar hacia ciertos niveles de intensidad de la experiencia emocional, generadas desde la propia dinámica del sujeto, no niegan la influencia que el medio externo pueda tener sobre la experiencia emocional del sujeto, sin embargo, sí califican esta influencia. Así, ante un mismo estímulo externo, el sujeto puede responder más o menos intensamente en un momento dado dependiendo de su nivel de activación emocional en ese momento. Por otro lado, ante un mismo estado de activación emocional, la magnitud de un cambio en la experiencia emocional va a depender de la magnitud del estímulo externo. De hecho, muchas veces los sujetos transitaron desde un estado de baja activación hacia estados de activación mucho más altos y viceversa. En estos casos, la aparición del estado de activación consecuente no es facilitada, e incluso puede ser inhibida, por el estado de activación antecedente del sujeto, lo que hace pensar, que el cónyuge debe haber jugado un rol preponderante en la existencia de estas transiciones.

Deben considerarse algunas limitaciones metodológicas y procedurales de este estudio. En primer lugar, el puntaje z para cada una de las transiciones fue calculado sobre la base de agregar los datos a través de los sujetos. Por lo tanto, las interpretaciones fueron realizadas en referencia a un sujeto abstracto que, quizás, no representa a ningún sujeto en particular o, a lo menos, a la mayor parte de los sujetos. Idealmente, sería necesario hacer el cálculo de estos puntajes en relación a cada individuo, lo que implica obtener secuencias de una longitud significativamente mayor a las que nosotros obtuvimos. En la práctica, no obstante, ello habría implicado un alto costo para las parejas participantes dado que se les solicitó que hicieran un reporte continuo de la intensidad de su experiencia, en varias emociones específicas, y en relación a una conversación altamente conflictiva. Para cumplir con este objetivo y, al mismo tiempo, obtener una secuencia suficientemente larga, habría sido necesario que el reporte se hiciera a intervalos sustantivamente menores que un minuto o, alternativamente, habría sido necesario que el periodo de interacción fuera mucho más prolongado. Una segunda consideración, atañe a la validez ecológica de los resultados de nuestro estudio. En la "vida real", las parejas no suelen acordar de antemano el tema de discusión, sino que éstos se originan de forma más espontánea. Tampoco es probable que discutan en ambientes que no les son familiares y, definitivamente, no lo hacen ante cámaras. Es posible que todos estos artificios de laboratorio conduzcan a que el grado de involucramiento emocional de las parejas sea menor al que habría en un contexto de interacción marital más natural. Una tercera limitación del estudio refiere a la posibilidad de inferir los resultados a la población. Aquí la dificultad no radica tanto en la posibilidad de generalizar las características de los participantes, puesto que el grupo era bastante heterogéneo ( en años de casados, nivel educacional, grado de ajuste marital, número de hijos), sino más bien, en el relativamente pequeño tamaño muestral (60 sujetos, 30 parejas).

Una promisoria línea de investigación a futuro sería relacionar el nivel de ajuste marital con el nivel de auto dependencia en la experiencia emocional versus su dependencia en relación al comportamiento del cónyuge. Dado lo planteado en la literatura, en el sentido de que las parejas insatisfechas presentan un mayor grado de dependencia afectiva respecto de los mensajes del cónyuge que las parejas satisfechas, uno esperaría que la auto dependencia en la experiencia emocional de estas últimas fuera mayor que en las primeras. 


\section{Referencias}

BAKeman, R., GotTMAn, J.M. (1986). Observing interaction: An introduction to sequential analysis. New York: Cambridge University Press.

Burman, B., Margolin, G., John, R.S. (1993). America's angriest home videos:

Behavioral contingencies observed in home reenactments of marital conflict. Journal of Consulting and Clinical Psychology. 61;28-39.

GotTMAn, J.M. (1998). Psychology and the study of marital processes. Annual Review of Psychology. 49;169-197.

Levenson, R.W., Carstensen, L.L., GotTMAN, J.M. (1994). The influence of age and gender on affect, physiology, and their interrelations: A study of long terms marriages. Journal of Personality and Social Psychology. 67;56-68

Levenson, R.W., GotTMAN, J.M. (1983). Marital interaction: Physiological linkage and affective exchange. Journal of Personality and Social Psychology. 45;587-597.

LOCKe, H.J., Wallace, K.M. (1959). Short marital adjustment and prediction test: Their reliability and validity. Marriage and Family Living. 21;251-255.

Margolin, G., Wampold, B.E. (1981). Sequential analysis of conflict and accord in distressed and nondistressed marital partners. Journal of Consulting and Clinical Psychology. 49;554-567.

Notarius, C.I., Benson, P.R., Sloane, D., VANZETTI, N.A. (1989). Exploring the interface between perception and behavior: An analysis of marital interaction in distressed and nondistressed couples. Behavioral Assessment. 11;39-64.

Weiss, R.L., Heyman, R.E. (1990). Observation of marital interaction. En: F.D.

Fincham, T.N. Bradbury, (Eds). The Psychology of Marriage: Basic issues and applications (87-117). New York: Guilford Press. 